\title{
Avaliação do modelo de predição clínica de Wells et al. no diagnóstico da trombose venosa profunda dos membros inferiores
}

\author{
Evaluation of a clinical prediction model by Wells et al. in the \\ diagnosis of deep venous thrombosis of the lower limbs
Veronica Barreto Fortes ${ }^{1}$, Hamilton Almeida Rollo ${ }^{2}$, Archangelo Tarciso Fortes Jr. ${ }^{1}$, Marcone de Lima Sobreira ${ }^{3}$, Fernanda Cardoso Santos ${ }^{4}$, Mariângela Giannini' ${ }^{2}$, Francisco Humberto de Abreu Maffei ${ }^{5}$

\section{Resumo}

Contexto: A aplicação de uma estratégia baseada em um modelo clínico associado ao mapeamento dúplex (MD) pode permitir um diagnóstico da trombose venosa profunda (TVP) mais seguro, eficaz e custo-efetivo.

Objetivo: Testar o modelo clínico de Wells et al. associado ao MD e verificar a ocorrência de TVP nos pacientes categorizados quanto à probabilidade de apresentar a doença, e determinar se, a partir dos resultados obtidos, seria possível reduzir o número de exames seriados com o MD.

Métodos: Os pacientes com suspeita clínica de TVP foram categorizados quanto à apresentação de TVP em baixa, moderada e alta probabilidade (BP, MP, AP) e, em seguida, submetidos ao MD. Pacientes com MD negativo repetiram o exame em $24-48$ horas e em 7 dias. Pacientes com exame positivo para TVP foram tratados. Todos os pacientes sem TVP foram convocados para reavaliação clínica em 3 meses.

Resultados: A ocorrência de TVP entre os 489 pacientes avaliados foi de $39,1 \%$ (191), sendo $35,6 \%$ identificados no exame inicial e $3,5 \%$ no exame seriado. Os índices de pacientes que apresentaram TVP foram de $6,1 \%$ no grupo de BP, $26,9 \%$ no grupo de MP e 79,5\% no grupo de AP. No exame seriado, o percentual de TVP foi de 2,4, 7,8 e 15,1\% nos grupos BP, MP e AP, respectivamente. Dos pacientes com MD negativo, $62,4 \%$ compareceram após 3 meses, e piora dos sintomas foi apresentada por apenas um paciente. Neste, o MD mostrou TVP de veia poplítea.

Conclusão: Os resultados obtidos sugerem que, para os pacientes com BP para TVP e MD negativo, seria possível prescindir do exame seriado, devido à baixa ocorrência de TVP neste grupo, tornando, assim, a abordagem diagnóstica mais simples.

Palavras-chave: Trombose venosa, diagnóstico, ultra-som.

\begin{abstract}
Background: The application of a diagnostic strategy based on a clinical model associated with duplex scanning (DS) may allow for a safer and more effective/cost-effective diagnosis of deep venous thrombosis (DVT).

Objective: To evaluate the clinical model proposed by Wells et al. associated with DS and verify the occurrence of DVT in patients divided into probability of presenting the disease, and assess the possibility of reducing the number of repeated DS based on the results obtained.

Methods: Suspected DVT patients were accordingly categorized into groups of low, moderate and high DVT probability (LP, MP and HP). The patients were then submitted to DS and those without DVT were rescheduled to repeat the examination in 24-48 hours and in 7 days. Patients positively diagnosed with DVT received proper treatment. All patients without DVT were summoned to return within 3 months.

Results: The incidence of DVT among all 489 patients was 39.1\% (191); of these, $35.6 \%$ were identified in the first examination and $3.5 \%$ in the follow-up. Among patients categorized as LP the occurrence was $6.1 \%$, $26.9 \%$ in the MP group and $79.5 \%$ in the HP group. On the follow-up exams the incidence of DVT in LP, MP and HP groups was 2.4, 7.8 e $13.2 \%$, respectively. Among patients with negative DS, $62.4 \%$ attended the reevaluation in 3 months and only one presented worsening of symptoms. This patient was then diagnosed with popliteal DVT using DS.

Conclusion: The results suggest that for patients with LP for DVT and negative DS, follow-up exams are not needed, since the occurrence of DVT was low in this group. This procedure simplifies the diagnostic process.
\end{abstract}

Keywords: Venous thrombosis, diagnosis, ultrasonics.

1. Mestre em Cirurgia em Bases Gerais da Cirurgia, Faculdade de Medicina de Botucatu (FMB), Universidade Estadual Paulista (UNESP), Botucatu, SP.

2. Doutor. Professor assistente, Disciplina de Cirurgia Vascular, FMB, UNESP, Botucatu, SP.

3. Médico, Serviço de Cirurgia Vascular, Hospital das Clínicas da FMB, UNESP, Botucatu, SP.

4. Biomédica. Ex-aprimoranda, Laboratório Vascular do Hospital das Clínicas da FMB, UNESP, Botucatu, SP.

5. Professor titular, Disciplina de Cirurgia Vascular, FMB, UNESP, Botucatu, SP.

Este trabalho foi apresentado no I Congresso Brasileiro de Eco-Doppler Vascular, realizado de 21 a 24 julho, 2004, em Vitória, ES.

Artigo submetido em 25.06.06, aceito em 30.01.07.

J Vasc Bras 2007;6(1):7-16.

Copyright $\odot 2007$ by Sociedade Brasileira de Angiologia e de Cirurgia Vascular. 


\section{Introdução}

A trombose venosa profunda (TVP) é uma afecção freqüente e potencialmente fatal. Estima-se que, nos EUA, a TVP ocorra em 1\% da população por ano, sendo a terceira doença cardiovascular mais comum ${ }^{1}$.

Sua complicação mais temida por sua morbimortalidade é a embolia pulmonar (EP), que pode ser evitada com o diagnóstico precoce e a instituição do tratamento anticoagulante. Outra complicação não letal, mas de forte impacto socioeconômico, é a síndrome pós-trombótica. Ela pode ocorrer a despeito do tratamento adequado da TVP, mas este, quando instituído precocemente, tende a minimizar seus efeitos.

O tratamento anticoagulante, apesar de ser altamente eficaz na prevenção da progressão da trombose, da embolização e da recorrência da doença, está associado a complicações e implica exames de sangue e visitas regulares ao médico, devendo ser instituído apenas após confirmação diagnóstica da TVP.

O diagnóstico clínico da TVP é impreciso, visto que, por um lado, apenas 20 a $40 \%$ dos pacientes com sintomas sugestivos têm a doença confirmada por exames objetivos. Por outro lado, 15 a 50\% dos casos de TVP não apresentam quadro clínico inicial característico. Sendo assim, seu diagnóstico de certeza se baseia em exames complementares.

Dentre os métodos diagnósticos disponíveis, o mapeamento dúplex (MD) é considerado o padrão-ouro dos exames não-invasivos, apresentando boa sensibilidade e especificidade para TVP proximal. No entanto, esta acurácia cai na TVP distal (nas veias da perna), com sensibilidade e especificidade em torno de $70 \%$ neste segmento ${ }^{2}$. O risco de embolia pulmonar decorrente de TVP isolada de veias da perna parece pequeno, mas existe um risco de progressão da trombose distal para segmentos proximais de até $20 \%{ }^{3}$. Por essa razão, alguns autores acreditam que, nos casos de MD negativo para TVP, este deve ser repetido no intervalo de 7 dias com o objetivo de detectar trombos em progressão $^{4-7}$.

Em uma tentativa de simplificar a abordagem diagnóstica, Wells et al. ${ }^{8}$ desenvolveram um modelo de predição clínica que classifica os pacientes quanto ao risco de apresentar TVP. Este modelo, associado aos exames complementares não-invasivos, mostrou-se útil em diversos estudos no procedimento diagnóstico da $\mathrm{TVP}^{3,8,9}$.

O objetivo deste trabalho foi testar o modelo de predição clínica de Wells et al. associado ao MD em pacientes com suspeita clínica de TVP atendidos no Hospital das Clínicas da Faculdade de Medicina de Botucatu e verificar: 1) a prevalência de TVP nos pacientes categorizados como alta, moderada e baixa probabilidade de apresentar a doença, e 2) se, a partir dos resultados obtidos, seria possível reduzir o número de exames seriados com o MD.

\section{Métodos}

O projeto de pesquisa foi aprovado pelo Comitê de Ética em Pesquisa da Faculdade de Medicina de Botucatu da Universidade Estadual Paulista (UNESP).

Os pacientes com suspeita clínica de TVP dos membros inferiores internados nas enfermarias e atendidos nas unidades de Pronto-Socorro ou de Triagem do Hospital das Clínicas da Faculdade de Medicina de Botucatu, UNESP foram avaliados prospectivamente pela aplicação do protocolo do estudo.

Foram incluídos pacientes com idade superior a 18 anos com suspeita de primeiro episódio de TVP, cujas queixas tivessem duração máxima de 30 dias. Estes foram incluídos mediante assinatura de um termo de consentimento livre e esclarecido.

Foram considerados critérios de exclusão: não concordar em participar do estudo, apresentar queixas com duração superior a 30 dias, apresentar sintomas ou sinais de embolia pulmonar, apresentar antecedente de TVP ou de embolia pulmonar, gravidez, ter idade inferior a 18 anos e/ou estar em uso de terapia anticoagulante.

Após a realização de anamnese, interrogatório sobre diversos aparelhos, antecedentes pessoais e familiares e exame físico geral e vascular, foi preenchido um protocolo baseado na proposta de Wells et al. ${ }^{8} \mathrm{O}$ paciente era instruído sobre o estudo e solicitado a ler e, em caso de 
concordância, assinar um termo de consentimento livre e esclarecido. As avaliações foram realizadas pelos médicos residentes da Disciplina de Cirurgia Vascular, do primeiro e segundo anos, ou pelo cirurgião vascular de plantão, que haviam sido previamente instruídos sobre o preenchimento do protocolo. Antes do início do trabalho, foi realizado um treinamento, durante 6 semanas, para o preenchimento do protocolo, e os autores verificaram e corrigiram os formulários preenchidos.

$\mathrm{O}$ protocolo incluiu perguntas aos pacientes ou acompanhantes sobre os fatores de risco para o desenvolvimento da doença tromboembólica venosa e os dados obtidos na realização do exame físico vascular (Tabela 1). Foi adotada a pontuação proposta por Wells et al., em que o paciente recebe 1 ponto para a presença de cada um dos fatores mostrados na Tabela 1. Além disso, foi investigada a presença de afecções outras que justificassem o quadro clínico. Caso o paciente apresentasse um diagnóstico diferencial mais provável, eram subtraídos 2 pontos de seu escore (Tabela 1).

De acordo com as pontuações obtidas neste protocolo, os pacientes foram classificados, quanto à probabilidade de apresentar TVP, em três grupos:
- Pacientes com baixa probabilidade (BP) de TVP: menor ou igual a 0 pontos.

- Pacientes com moderada probabilidade (MP) de TVP: 1 a 2 pontos.

- Paciente com alta probabilidade (AP) de TVP: 3 ou mais pontos.

Em seguida, os pacientes eram encaminhados ao Laboratório de Métodos Não-Invasivos da Cirurgia Vascular para realização do MD. Aqueles com resultado positivo para TVP foram tratados com terapêutica adequada. Nos casos em que o resultado foi negativo ou duvidoso para TVP, o exame foi repetido em 24-48 horas e após 7 dias. Caso persistisse dúvida, o paciente era submetido à flebografia. Os pacientes com exames seqüenciais negativos foram orientados a informar a equipe médica no caso de apresentarem sinais ou sintomas sugestivos de TVP ou EP e a retornar em 3 meses para reavaliação clínica (Figura 1). O MD foi realizado no Laboratório Vascular utilizando-se o equipamento Platinum Philips CVI (Color Velocity Imaging), com transdutor de freqüência de 7,5 $\mathrm{MHz}$. Quando necessário, em pacientes mais obesos e em veias mais profundas, foi utilizado transdutor de $3 \mathrm{MHz}$,

Tabela 1 - Modelo de predição clínica proposto por Wells et al. ${ }^{10}$

\begin{tabular}{lc}
\hline Características clínicas & Escore \\
\hline Câncer em atividade & 1 \\
Paresia, paralisia, ou imobilização com gesso dos membros inferiores & 1 \\
Imobilização (> 3 dias) ou cirurgia maior (até 4 semanas) & 1 \\
Aumento da sensibilidade ao longo das veias do sistema venoso profundo & 1 \\
Edema em todo o membro & 1 \\
Edema da panturrilha (> 3 cm) em relação à perna normal & 1 \\
Edema depressível (cacifo) maior na perna afetada (unilateral) & 1 \\
Veias colaterais superficiais & 1 \\
Diagnóstico diferencial mais provável (celulite, tromboflebite superficial, \\
alterações osteoarticulares, câimbras, ruptura muscular ou tendínea, alterações
\end{tabular}




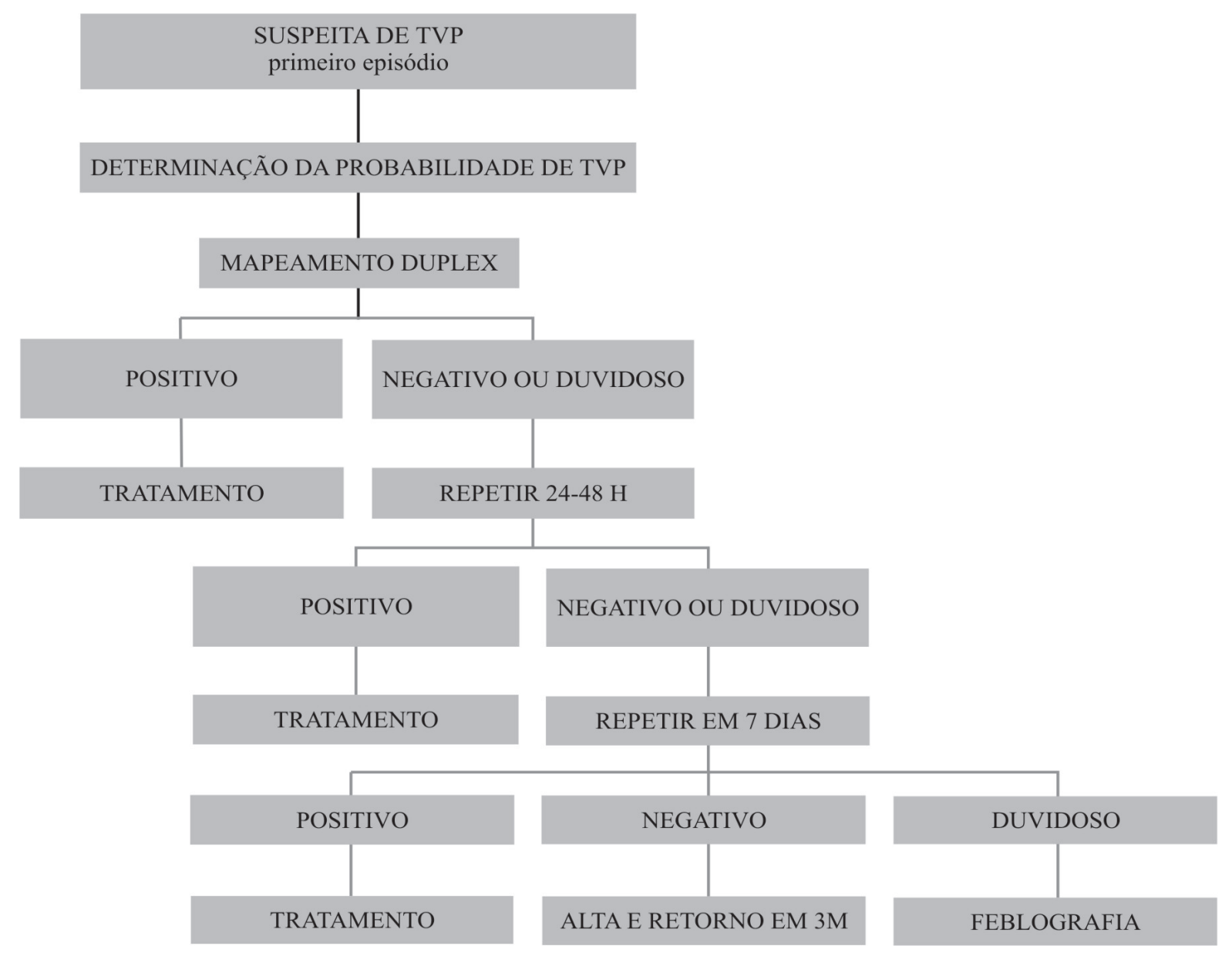

$\mathrm{TVP}=$ trombose venosa profunda

Figura 1 - Algoritmo resumindo a abordagem diagnóstica para pacientes com suspeita de TVP

visto que estes eram os transdutores disponíveis no Laboratório. Os exames foram realizados por cirurgiões vasculares habilitados ou pelos residentes da cirurgia vascular, sob a supervisão daqueles. Todos os exames foram checados por profissional com título de especialista em ultra-sonografia vascular.

O MD venoso foi realizado com o paciente em decúbito dorsal horizontal em proclive. Foi realizado um mapeamento das veias profundas em toda a sua extensão em corte transversal, observando sua morfologia, distribuição anatômica, compressibilidade e a presença de fluxo venoso. Foram avaliadas as veias ilíaca externa, femoral comum, femoral superficial, poplítea, fibulares e tibiais posteriores. Para avaliação das veias poplítea e fibulares, foi adotado o decúbito ventral horizontal ou lateral com discreta flexão do joelho.

Foram utilizados como critérios para o diagnóstico o teste de compressibilidade, a verificação do fluxo venoso e suas alterações e, quando possível, a visualização de material ecogênico, sugestivo de trombo, no interior das veias. A TVP foi considerada proximal quando envolvia veia poplítea e/ou veias mais proximais (ilíaca, femoral comum e femoral superficial) e distal quando era restrita às veias da perna (fibulares e/ou tibiais posteriores).

Os pacientes que retornaram após 3 meses foram reavaliados clinicamente e interrogados sobre melhora 
dos sintomas e sinais de TVP e sobre queixas relacionadas à doença tromboembólica. Em seguida, foi realizado um exame físico vascular e, em caso de agravamento dos sintomas e sinais, foi realizado novo MD.

$\mathrm{Na}$ análise estatística, utilizou-se, para comparação das proporções envolvendo duas populações, a distribuição normal e, para os casos de mais de duas populações, o método de Tukey. Nos casos de proporções multinomiais, utilizou-se o teste de Goodman ${ }^{9}$. O nível de significância considerado foi de 5\% ( $<<0,05)$.

\section{Resultados}

Foram avaliados 489 pacientes consecutivos que preenchiam os critérios de inclusão do estudo no período de agosto de 2000 a dezembro de 2002. Eram 216 pacientes do sexo masculino (44,2\%) e 273 do sexo feminino (55,8\%), com idades entre 18 e 96 anos e média de idade de 57,1 anos. Dos pacientes avaliados, 291 $(59,3 \%)$ eram ambulatoriais e $198 \quad(41,7 \%)$ encontravam-se internados, incluindo pacientes clínicos e cirúrgicos, independentemente do tempo de internação.

Foram encontrados 147 (30\%) pacientes com BP, $171(35 \%)$ com MP e $171(35 \%)$ com AP.

A TVP ocorreu em 191 pacientes (39,1\%), sendo que a TVP nos grupos de BP, MP e AP apareceu na ordem de 6,1\% (9), 26,9\% (46) e 79,5\% (136), respectivamente. A diferença é estatisticamente significante (Tabela 2). A ocorrência foi maior entre os homens $(44,9 \%) \mathrm{em}$ relação às mulheres $(34,4 \%)$, sendo essa diferença estatisticamente significativa.
$\mathrm{Na}$ comparação entre pacientes internados $(40,7 \%)$ e ambulatoriais $(38,1 \%)$ com TVP, não foi encontrada diferença significativa. Entre os pacientes ambulatoriais, a ocorrência de TVP nos grupos de BP, MP e AP foi de 5,6, 31,2 e 86,2\%, respectivamente. Entre os pacientes internados, essa ocorrência foi de 7,7\% no grupo de BP, de $21,3 \%$ no de MP e de $72,6 \%$ no de AP. Ao se fazer a comparação da ocorrência de TVP entre os pacientes ambulatoriais e internados, foi encontrada diferença estatística entre os pacientes com AP, com predomínio de TVP entre os pacientes ambulatoriais (Tabela 3).

Foi observada maior ocorrência de TVP proximal em relação à TVP distal nos grupos de MP e AP. Entre os pacientes com BP, não houve diferença estatisticamente significante. $O$ percentual de TVP proximal não diferiu entre os grupos de BP e MP (55,6 e 69,6\%), mas foi superior a estes no grupo de AP com 90,4\% (Tabela 4).

Dentre os 298 pacientes com MD inicial negativo, $273(91,6 \%)$ retornaram para realizar os exames seriados, sendo 124 (87,9\%) pacientes do grupo com BP, 116 $(92,8 \%)$ daqueles com MP e 33 (95\%) dos pacientes com AP. Houve diferença estatisticamente significativa quando comparado o número de retornos no grupo de $\mathrm{BP}$ com o número de retornos nos grupos de MP e AP. Foi diagnosticada TVP em 8,9\% (17) destes pacientes, sendo 2,4\% (3) no grupo de BP, 7,8\% (9) no grupo de MP e $15,1 \%$ (5) no de AP. Houve diferença estatística para a ocorrência entre os três grupos (Tabela 5). Dois pacientes foram a óbito antes de completar a investigação por causa provavelmente não relacionada a TVP

Tabela 2 - Distribuição de freqüências absolutas e relativas dos pacientes segundo a classificação e a presença de TVP

\begin{tabular}{|c|c|c|c|c|c|c|}
\hline \multirow[b]{2}{*}{ Probabilidade } & \multicolumn{2}{|c|}{ Com TVP } & \multicolumn{2}{|c|}{ Sem TVP } & \multicolumn{2}{|c|}{ Total } \\
\hline & $\mathbf{n}$ & $\%$ & $\mathbf{n}$ & $\%$ & $\mathbf{n}$ & $\%$ \\
\hline $\mathrm{BP}$ & 9 & $6,1^{\mathrm{a} *}$ & 138 & 93,9 & 147 & 100,0 \\
\hline MP & 46 & $26,9^{b}$ & 125 & 73,1 & 171 & 100,0 \\
\hline AP & 136 & $79,5^{\mathrm{c}}$ & 35 & 20,5 & 171 & 100,0 \\
\hline
\end{tabular}

* Percentuais seguidos de diferentes letras diferem estatisticamente entre si $(\mathrm{p}<0,05)$.

$\mathrm{AP}=$ alta probabilidade; $\mathrm{BP}=$ baixa probabilidade; $\mathrm{MP}=$ moderada probabilidade; $\mathrm{TVP}=$ trombose venosa profunda. 
Tabela 3 - Distribuição de freqüências absolutas e relativas dos pacientes segundo a classificação, a presença de TVP e o local da avaliação

\begin{tabular}{|c|c|c|c|c|c|c|c|c|c|c|c|c|}
\hline \multirow[b]{3}{*}{ Probabilidade } & \multicolumn{4}{|c|}{ Sem TVP } & \multicolumn{4}{|c|}{ Com TVP } & \multicolumn{4}{|c|}{ Total } \\
\hline & \multicolumn{2}{|c|}{ Ambulatorial } & \multicolumn{2}{|c|}{ Internado } & \multicolumn{2}{|c|}{ Ambulatorial } & \multicolumn{2}{|c|}{ Internado } & \multicolumn{2}{|c|}{ Ambulatorial } & \multicolumn{2}{|c|}{ Internado } \\
\hline & n & $\%$ & $\mathbf{n}$ & $\%$ & $\mathbf{n}$ & $\%$ & $\mathbf{n}$ & $\%$ & $\mathbf{n}$ & $\%$ & n & $\%$ \\
\hline $\mathrm{BP}$ & 102 & 94,4 & 36 & 92,3 & 6 & $5,6^{\mathrm{a} *}$ & 3 & $7,7^{\mathrm{a}}$ & 108 & 100,0 & 39 & 100,0 \\
\hline MP & 66 & 68,8 & 59 & 78,7 & 30 & $31,2^{\mathrm{b}}$ & 16 & $21,3^{\mathrm{b}}$ & 96 & 100,0 & 75 & 100,0 \\
\hline AP & 12 & 13,8 & 23 & 27,4 & 75 & $86,2^{\mathrm{c}}$ & 61 & $72,6^{\mathrm{d}}$ & 87 & 100,0 & 84 & 100,0 \\
\hline
\end{tabular}

* Percentuais seguidos de diferentes letras diferem estatisticamente entre si $(\mathrm{p}<0,05)$.

$\mathrm{AP}=$ alta probabilidade; $\mathrm{BP}=$ baixa probabilidade; $\mathrm{MP}=$ moderada probabilidade; $\mathrm{TVP}=$ trombose venosa profunda.

Tabela 4 - Distribuição de freqüências absolutas e relativas dos pacientes segundo a classificação, a presença de TVP e o nível da TVP

\begin{tabular}{|c|c|c|c|c|}
\hline \multirow[b]{2}{*}{ Probabilidade } & \multicolumn{2}{|c|}{ TVP proximal } & \multicolumn{2}{|c|}{ TVP distal } \\
\hline & $\mathbf{n}$ & $\%$ & $\mathbf{n}$ & $\%$ \\
\hline $\mathrm{BP}$ & 5 & $55,6^{\mathrm{a} *}$ & 4 & $44,4^{\mathrm{a}}$ \\
\hline MP & 32 & $69,6^{\mathrm{a}}$ & 14 & $30,4^{\mathrm{c}}$ \\
\hline AP & 123 & $90,4^{\mathrm{b}}$ & 13 & $9,6^{\mathrm{d}}$ \\
\hline
\end{tabular}

* Percentuais seguidos de diferentes letras diferem estatisticamente entre si $(p<0,05)$.

$\mathrm{AP}=$ alta probabilidade; $\mathrm{BP}=$ baixa probabilidade; $\mathrm{MP}=$ moderada probabilidade; $\mathrm{TVP}=$ trombose venosa profunda.

(um acidente vascular cerebral e um traumatismo craniencefálico). No entanto, não foi autorizada necropsia.

Dos 298 pacientes sem TVP detectada nos exames seriados, $186(62,4 \%)$ compareceram ao retorno em 3 meses. O percentual de complicações tromboembólicas foi de $0,5 \%$. Dos 186 pacientes, 47,8\% eram do grupo de $\mathrm{BP}$, e nenhum deles apresentava queixas sugestivas de doença tromboembólica. O percentual de retorno nos grupos de MP e AP diferiram estatisticamente do grupo de BP. Dos pacientes com MP, 77,6\% retornaram e um paciente apresentava piora dos sintomas e sinais. Foi realizado MD que mostrou TVP de veias poplítea e tibiais posteriores. Os demais pacientes não apresentavam queixas. $\mathrm{O}$ índice de retornos no grupo de AP foi estatisticamente semelhante ao de MP com 65,7\%, e nenhum dos pacientes apresentava queixas compatíveis com TVP ou EP (Tabela 6). Neste grupo, quatro pacientes foram a óbito antes da avaliação. Dois pacientes por sépsis, um por neoplasia maligna e um por infecção oportunista devido à infecção por HIV. Não foi realizada necropsia em nenhum dos casos. Portanto, não foi possível excluir doença tromboembólica.

\section{Discussão}

Encontramos uma distribuição semelhante dos 489 pacientes incluídos no estudo entre os grupos de BP, MP e AP, com 30, 35 e 35\%, respectivamente. Essa distribuição difere da relatada por Wells et al. em seus trabalhos de 1995 e 1997, em que predominaram pacientes com BP, com 55,5 e 57\%, respectivamente ${ }^{10,11}$. Wells et al. não incluíram pacientes internados em sua amostra, o 
que poderia explicar a diferença na distribuição. No entanto, mesmo quando consideramos apenas os pacientes ambulatoriais de nossa amostra, embora encontremos discreto predomínio de pacientes com BP (37\%), em relação aos grupos MP (33\%) e AP (30\%), não houve diferença estatística. Isso poderia ser explicado pelo fato de, em nosso meio, os pacientes chegarem mais tardiamente para avaliação, quando os sinais e sintomas são mais importantes. Esse fato pode refletir um atraso no encaminhamento dos pacientes pelos médicos generalistas ou uma tendência dos pacientes de procurarem o médico apenas quando o quadro clínico já está avançado.

A ocorrência total de TVP na amostra foi de 39,1\%, que diferiu de diversos estudos ${ }^{10-15}$. Foi de $38,1 \%$ entre os pacientes ambulatoriais e de $40,7 \%$ entre os internados. Seria esperada maior prevalência entre os pacientes internados quando comparados aos ambulatoriais. No entanto, isso não foi observado, provavelmente devido ao fato de nossa amostra de pacientes ambulatoriais não excluir aqueles que estiveram recentemente internados ou provenientes de casas de repouso, tendo em vista que estas informações não foram abordadas na avaliação inicial. Heit et al. ${ }^{16}$, em trabalho retrospectivo avaliando a incidência de TVP entre pacientes de uma comunidade, referiram ocorrência 100 vezes maior de TVP em pacientes hospitalizados, incluindo sintomáticos e assintomáticos, quando comparados com a população geral.

Um estudo anterior em nosso serviço realizado por Rollo et al. ${ }^{17}$ encontrou $51,6 \%$ de TVP entre 658 pacientes com suspeita clínica de TVP. Essa incidência superior pode ser explicada por um uso mais criterioso dos exames complementares, sendo excluídos da execução do MD pacientes com outras afecções que justificassem os sintomas e sinais.

Tabela 5 - Distribuição de freqüências absolutas e relativas dos pacientes segundo a classificação, o retorno para exame seriado e a presença de TVP no exame seriado

\begin{tabular}{|c|c|c|c|c|}
\hline \multirow[b]{2}{*}{ Probabilidade } & \multicolumn{2}{|c|}{ Retorno p/ exame seriado } & \multicolumn{2}{|c|}{ TVP no exame seriado } \\
\hline & $\mathbf{n}$ & $\%$ & $\mathbf{n}$ & $\%$ \\
\hline $\mathrm{BP}$ & 124 & $87,9^{\mathrm{a} *}$ & 3 & $2,4^{\mathrm{c} *}$ \\
\hline MP & 116 & $92,8^{\mathrm{b}}$ & 9 & $7,8^{\mathrm{d}}$ \\
\hline AP & 33 & $95^{\mathrm{b}}$ & 5 & $15,1^{\mathrm{e}}$ \\
\hline
\end{tabular}

* Percentuais seguidos de diferentes letras diferem estatisticamente entre si $(p<0,05)$.

$\mathrm{AP}=$ alta probabilidade; $\mathrm{BP}=$ baixa probabilidade; $\mathrm{MP}=$ moderada probabilidade; $\mathrm{TVP}=$ trombose venosa profunda.

Tabela 6 - Distribuição de freqüências absolutas e relativas dos pacientes segundo a classificação e o retorno em 3 meses e complicações presentes no retorno

\begin{tabular}{|c|c|c|c|c|}
\hline \multirow[b]{2}{*}{ Probabilidade } & \multicolumn{2}{|c|}{ Retorno em 3 meses } & \multicolumn{2}{|c|}{ Complicações } \\
\hline & $\mathbf{n}$ & $\%$ & $\mathbf{n}$ & $\%$ \\
\hline $\mathrm{BP}$ & 66 & $47,8^{\mathrm{a} *}$ & 0 & $0^{\mathrm{c}}$ \\
\hline MP & 97 & $77,6^{\mathrm{b}}$ & 1 & $1,1^{\mathrm{c}}$ \\
\hline AP & 23 & $65,7^{\mathrm{b}}$ & 0 & $0^{\mathrm{c}}$ \\
\hline
\end{tabular}

* Percentuais seguidos de diferentes letras diferem estatisticamente $(\mathrm{p}<0,05)$.

$\mathrm{AP}=$ alta probabilidade; $\mathrm{BP}=$ baixa probabilidade; $\mathrm{MP}=$ moderada probabilidade; $\mathrm{TVP}=$ trombose venosa profunda . 
Wells et al. ${ }^{10,11}$ encontraram prevalência de $25,5 \mathrm{e}$ $16 \%$, respectivamente entre pacientes ambulatoriais. Anderson et al. ${ }^{8}$, avaliando pacientes no prontosocorro com suspeita de TVP, encontraram prevalência de $13,1 \%$. Miron et al. ${ }^{14}$ encontraram $21,1 \%$ de casos de TVP entre 270 pacientes ambulatoriais.

Em trabalho retrospectivo avaliando 540 pacientes com suspeita de TVP submetidos ao MD, Mattos et al. ${ }^{18}$ encontraram prevalência de $24 \%$ de TVP, sendo que os trombos eram restritos às veias da perna em 33\% dos casos. Em nossa casuística, dentre os 191 casos de TVP, 18,8\% eram limitados ao segmento distal, 44,4\% no grupo de BP, 30,4\% no grupo de MP e $9,6 \%$ no de AP. Houve diferença estatisticamente significante entre o grupo de $\mathrm{AP}$ e os grupos de $\mathrm{MP}$ e $\mathrm{BP}$, os quais, no entanto, não diferiram entre si, o que mostra a correlação da extensão da trombose com a intensidade do quadro clínico.

Souza $^{19}$, em estudo utilizando a flebografia, relata $25 \%$ de TVP no grupo de BP, $50 \%$ no de MP e $85 \%$ no de AP. Dentre os pacientes com TVP, encontrou TVP proximal em $12,5 \%$ no grupo de $\mathrm{BP}$, em $25 \%$ no grupo de MP e em 80,6\% no grupo de AP. No presente estudo, encontramos, entre os pacientes com TVP, acometimento proximal em $91,1 \%$ dos pacientes, sendo 55,6\% entre os pacientes classificados como BP, $69,6 \%$ nos de MP e $90,4 \%$ nos de AP (Tabela 4 ). Souza ${ }^{19}$ pode ter encontrado maior freqüência de TVP nos grupos de BP e MP do que o observado no presente trabalho possivelmente por ter utilizado a flebografia como método diagnóstico, que é um exame mais sensível do que o MD. Isso também explicaria por que encontrou maior ocorrência de TVP distal do que o observado neste trabalho.

A ocorrência de TVP nos grupos de BP, MP e AP foi de $6,1,26,9$ e 79,5\%, respectivamente. Foi de 5,6, 31,2 e $86,2 \%$ entre os pacientes ambulatoriais e de 7,7, 21,3 e $72,6 \%$ entre os internados. Os achados confirmam os de Wells et al. ${ }^{10,11}$ que, em seus estudos com pacientes ambulatoriais, encontraram, em 1995, prevalência de 5, 33 e $85 \%$ de TVP nos grupos de BP, MP e AP, e, em 1997, encontraram 3,17 e 75\%, respectivamente. Anderson et al. ${ }^{8}$ encontraram proporções semelhantes para os grupos de BP e MP, com 3,2 e 14,3\%, respectivamente, e maior prevalência de TVP no grupo de AP (49\%).

Está bem estabelecido que os pacientes com suspeita de TVP devem ser submetidos ao MD ou a outro exame complementar disponível, preferencialmente não-invasivo $^{20,21}$. O MD está disponível na maioria dos serviços, e trata-se de exame de fácil execução, que não provoca desconforto para o paciente. Entretanto, para os pacientes com MD negativo, permanece a dúvida, uma vez que 10 a $20 \%$ dos pacientes com TVP sintomática têm TVP isolada de perna, e esta pode passar despercebida no $\mathrm{MD}^{21}$; pelo menos 20 a $30 \%$ desses trombos irão progredir proximalmente, aumentando o risco de embolia pulmonar ${ }^{3,22-24}$. Por isso, se propõe a realização do exame seriado quando o MD é negativo nos pacientes sintomáticos para se detectar esses casos ${ }^{25}$. Em nosso estudo, foi realizado exame seriado em $91,6 \%$ dos pacientes com exame inicial negativo. O maior número de retornos foi no grupo de AP $(95 \%)$, provavelmente por tratar-se de pacientes mais sintomáticos. Nos demais grupos, foi necessário realizar busca ativa em grande número de casos, principalmente entre os pacientes de BP.

A prevalência de TVP no exame seriado foi de $8,9 \%$, sendo $2,4 \%$ no grupo de BP, 7,8\% no grupo de MP e $15,1 \%$ no grupo de AP. É importante ressaltar que os três pacientes do grupo de BP que apresentaram exame seriado positivo também apresentaram agravamento dos sintomas, sugerindo que os pacientes com BP poderiam prescindir deste exame, exceto nos casos de piora do quadro clínico. Assim, seria possível reduzir em torno de $29 \%$ os exames de MD.

Wells et al. ${ }^{10}$, avaliando 593 pacientes com suspeita de TVP, realizaram exame seriado em 7 dias apenas nos pacientes com MP. Pacientes com AP e MD negativo foram submetidos à flebografia, o mesmo acontecendo aos pacientes com BP e MD positivo. Observaram 1,8\% de TVP no MD seriado. A técnica utilizada para a avaliação do sistema venoso profundo nos trabalhos de Wells et al. difere da realizada em nosso serviço. Aqueles autores avaliam apenas as veias proximais até o início da 
trifurcação da veia poplítea, não sendo avaliadas as veias da perna. Isso poderia explicar, em parte, a causa de ter sido encontrado maior número de casos de TVP no exame seriado no presente trabalho. Entre os pacientes com AP e MD negativo, Wells et al. encontraram TVP em 23,5\% das flebografias. No acompanhamento de 3 meses dos pacientes sem TVP, ocorreram apenas três eventos tromboembólicos $(0,6 \%)$, sendo um entre os pacientes de BP e dois entre os de MP.

Encontramos resultados semelhantes durante o acompanhamento de nossos pacientes, mas o número de retornos em 3 meses foi menor do que os retornos para MD seriado. Dos 186 pacientes $(62,4 \%)$ que retornaram, apenas um, no grupo de MP, apresentava agravamento das queixas iniciais com aparecimento de novos sintomas. Ele foi submetido a novo MD, que mostrou TVP de veia poplítea e veias tibiais posteriores. Nenhum paciente retornou com queixas sugestivas de EP. O índice de complicações tromboembólicas foi baixo $(0,5 \%)$ no período de 3 meses de seguimento, não se justificando esse tipo de acompanhamento para a maioria dos pacientes.

Anderson et al. ${ }^{8}$, utilizando a mesma abordagem de Wells et al., encontraram $1,1 \%$ de TVP no exame seriado e, no grupo de AP com MD negativo, 13,8\% apresentaram TVP na flebografia. Um paciente no grupo de BP apresentou piora dos sintomas e sinais e foi submetido ao MD em 7 dias, sendo diagnosticada TVP de perna. Estes autores observaram $0,7 \%$ de complicações tromboembólicas no seguimento de 3 meses, resultado semelhante ao encontrado no presente estudo.

A prevalência de TVP nos grupos de BP, MP e AP correspondeu às expectativas, ou seja, foi baixa no grupo de BP e alta no grupo de AP.

Em conclusão, os resultados obtidos no presente trabalho sugerem que o modelo de predição clínica de Wells et al. associado ao MD pode facilitar a estratégia diagnóstica da TVP. Sugerem também que, nos pacientes categorizados com BP e MD negativo, não é necessário realizar o MD seriado, já que, nesse grupo, a prevalência de TVP durante o seguimento foi baixa.
Deste modo, o exame deve ser repetido apenas quando há piora dos sintomas e sinais.

\section{Referências}

1. Hirsh J, Hoak J. Management of deep vein thrombosis and pulmonary embolism. A statement for healthcare professionals. Council on Thrombosis (in consultation with the Council on Cardiovascular Radiology), American Heart Association. Circulation. 1996;93:2212-45.

2. Hirsh J, Raschke R, Warkentin TE, Dalen JE, Deykin D, Poller L. Heparin: mechanism of action, pharmacokinetics, dosing considerations, monitoring, efficacy and safety. Chest. 1995;108(4 Suppl):258S-75S.

3. Cogo A, Lensing AW, Prandoni P, Hirsh J. Distribution of thrombosis in patients with symptomatic deep vein thrombosis. Implications for simplifying the diagnostic process with compression ultrasound. Arch Intern Med. 1993;153:2777-80.

4. Cogo A, Lensing AW, Koopman MM, et al. Compression ultrasonography for diagnostic management of patients with clinically suspected deep vein thrombosis: prospective cohort study. BMJ. 1998;316:17-20.

5. Kearon C, Julian JA, Newman TE, Ginsberg JS. Noninvasive diagnosis of deep vein thrombosis. McMaster Diagnostic Imaging Practice Guidelines Initiative. Ann Intern Med. 1998;128:663-77.

6. Wolf B, Nichols DM, Duncan JL. Safety of a single duplex scan to exclude deep venous thrombosis. Br J Surg. 2000;87:1525-8.

7. Hirsh J, Lee A. How we diagnose and treat deep vein thrombosis. Blood 2002;99:3102-10.

8. Anderson DR, Wells PS, Stiell I, et al. Thrombosis in the emergency department: use of a clinical diagnosis model to safely avoid the need for urgent radiological investigation. Arch Intern Med. 1999;159:477-82.

9. Tick LW, Ton E, van Voorthuizen T, et al. Practical diagnostic management of patients with clinically suspected deep vein thrombosis by clinical probability test, compression ultrasonography, and D-dimer test. Am J Med. 2002;113:630-5.

10. Wells PS, Anderson DR, Bormanis J, et al. Value of assessment of pretest probability of deep-vein thrombosis in clinical management. Lancet. 1997;350:1795-8.

11. Zar JH. More on dichotomous variables. In: Zar JH. Biostatistical analysis. 4th ed. Upper Saddle River: Prentice Hall; 1999. p. 516-65.

12. Wells PS, Hirsh J, Anderson DR, et al. Accuracy of clinical assessment of deep-vein thrombosis. Lancet. 1995;345:1326-30.

13. Perrier A, Desmarais S, Miron MJ, et al. Non-invasive diagnosis of venous thromboembolism in outpatients. Lancet. 1999;353:190-5.

14. Miron MJ, Perrier A, Bounameaux H. Clinical assessment of suspected deep vein thrombosis: comparison between a score and empirical assessment. J Intern Med. 2000;247:249-54. 
15. Dryjski M, O'Brien-Irr MS, Harris LM, Hassett J, Janicke D. Evaluation of a screening protocol to exclude the diagnosis of deep vein thrombosis among emergency department patients. J Vasc Surg. 2001;34:1010-5.

16. Heit JA, Melton LJ 3rd, Lohse CM, et al. Incidence of venous thromboembolism in hospitalized patients vs community residents. Mayo Clin Proc. 2001;76:1102-10.

17. Rollo HA, Lastoria S, Yoshida WB, et al. Mapeamento dúplex no diagnóstico da trombose venosa profunda dos membros inferiores. In: Programa Oficial do 32 Congresso Brasileiro de Angiologia e Cirurgia Vascular; 1997, Curitiba. 120.

18. Mattos MA, Melendres G, Sumner DS, et al. Prevalence and distribution of calf vein thrombosis in patients with symptomatic deep venous thrombosis: a color-flow duplex study. J Vasc Surg. 1996;24:738-44.

19. Souza E. Validação de modelo de predição clínica para o diagnóstico de trombose venosa profunda dos membros inferiores [dissertação]. São Paulo: Universidade Federal de São Paulo; 2001.

20. Haeger K. Problems of acute deep venous thrombosis. I. The interpretation of signs and symptoms. Angiology. 1969;20:219-23.

21. Richards KL, Armstrong JD Jr., Tikoff G, Hershgold EJ, Booth JL, Rampton JB. Noninvasive diagnosis of deep-vein thrombosis. Arch Intern Med. 1976;136:1091-6.
22. Wells PS, Hirsh J, Anderson DR, et al. A simple clinical model for the diagnosis of deep vein thrombosis combined with impedance plethysmography: potential for an improvement in the diagnostic process. J Intern Med. 1998:243:15-23.

23. Kakkar VV, Howe CT, Flanc C, Clarke MB. Natural history of postoperative deep-vein thrombosis. Lancet. 1969;2:230-2.

24. Lagerstedt CI, Olsson CG, Fagher BO, Oqvist BW, Albrechtsson U. Need for long-term anticoagulant treatment in symptomatic calf-vein thrombosis. Lancet. 1985;2:515-8.

25. Cogo A, Lensing AW, Prandoni P, Hirsh J. Distribution of thrombosis in patients with symptomatic deep vein thrombosis. Implications for simplifying the diagnostic process with compression ultrasound. Arch Intern Med. 1993;153:2777-80.

Correspondência:

Veronica Barreto Fortes

Rua Deoclides Freire, 227, Vila Santista

CEP 12941-060 - Atibaia, SP

Tel.: (11) 4402.3275, (11) 9689.8654

E-mail: bvfortes@terra.com.br 